\title{
la mariposa y el violín \\ la urgencia de una cita: infancia(s), escuela(s) e igualdad
}

resumen

patricia raquel redondo ${ }^{1}$ universidad nacional de la plata, argentina

Este trabajo presenta una reflexión política pedagógica sobre la educación con infancias populares. Parte de su contenido refiere a un trabajo de investigación que intenta dilucidar si en la Argentina la escuela pública inscripta en territorios marcados traumáticamente por la desigualdad puede construir otras experiencias educativas que alteren el orden de lo dado. Se intenta explorar a partir de una escena escolar la posibilidad de pensar la gestualidad escolar desde el movimiento y la variación que se abre cuando un colectivo docente disputa los sentidos de la igualdad y construye otros modos de ver la infancia, las infancias. Es un texto que explora condiciones de posibilidad ante el avance brutal del neoliberalismo en Argentina. Los niños y niñas de sectores populares son víctimas del gatillo fácil, la represión y la persecución de las fuerzas de seguridad; sus trayectorias educativas se ven interrumpidas con frecuencia por factores ligados al agravamiento de sus condiciones de vida. La infantilización de la pobreza en los países latinoamericanos se refleja en el terreno educativo. Las poblaciones infantiles segregadas asisten a circuitos escolares devaluados que reproducen los procesos de exclusión. Este trabajo intenta recuperar las experiencias educativas que interrumpen lo esperado, desarticulan el discurso del miedo y la inseguridad y desafían la equivalencia discursiva que asocia a un niño o una niña pobre con un peligro social. El propósito es poner en discusión que la gestualidad, el accionar institucional anclado en las preguntas y el movimiento que propicia el pensamiento permiten imaginar otros mundos posibles, escribir otras melodías con variaciones a las esperadas e inventar otras texturas polifónicas en educación. Es decir, atender aquellos pequeños movimientos que en clave de igualdad pueden en la vida de los sujetos definir una dirección u otra. La escuela de sectores populares con frecuencia se encuentra atrapada en procesos muy complejos; sin embargo, son innumerables las acciones que desde la educación se pueden llevar adelante para disputar los sentidos de la enseñanza en términos de lo público.

palabras clave: escuela(s); educar; infancia(s); igualdad.

\section{a borboleta e o violino a urgência de um encontro: infância(s), escola(s) e igualdade.}

resumo

Este trabalho apresenta uma reflexão político-pedagógica sobre a educação com infâncias das classes populares. Parte do seu conteúdo se refere a um trabalho de pesquisa que tenta elucidar se na Argentina, a escola pública inscrita em territórios apontados traumaticamente pela desigualdade, consegue construir outras experiências educativas que transformem a ordem do estabelecido. A partir de uma cena escolar, tenta-se analisar a possibilidade de pensar a gestualidade do movimento e a variação que se abre quando o conjunto dos docentes disputa os sentidos da igualdade e constrói outros modos de ver a infância, as infâncias. É um texto que explora condições de possibilidade diante do avanço brutal do

${ }^{1}$ E-mail: redpatricia@gmail.com 
neoliberalismo na Argentina. Vítimas do "gatilho fácil", da repressão e da persecução das forças de segurança, as crianças das classes populares acabam vendo como suas trajetórias educativas são interrompidas com frequência por fatores ligados ao agravamento de suas condições de vida. A infantilização da pobreza nos países latino-americanos se reflete no terreno educativo. As populações infantis segregadas vivenciam ciclos escolares desvalorizados que reproduzem os processos de exclusão. Desse modo, esse trabalho tenta recuperar as experiências educativas que interrompem o esperado; essas que desarticulam o discurso do medo, a falta de segurança e desafiam a equivalência discursiva que associa uma criança pobre ao perigo social. O propósito aqui é colocar em discussão como a gestualidade, a ação institucional, ancorada nas perguntas e o movimento que propicia o pensamento, permitem imaginar outros mundos possíveis, escrever outras melodias com variações diferentes às ansiadas e inventar outras texturas polifônicas em educação. Isto é, atender aos pequenos movimentos que em clave de igualdade podem, na vida dos sujeitos, definirem um caminho diferente. A escola das classes populares com frequência se descobre capturada em processos muito complexos. No entanto, são inúmeras as ações que da educação podem ser levadas adiante para discutir os sentidos do ensino em termos do público.

palavras-chave: escola(s); educar; infância(s); igualdade.

\section{the butterfly and the violin the urgency of an appointment: childhood(s), school(s) and inequality}

\section{abstract}

This paper presents a political and pedagogical reflection on the education of the workingclass childhoods. Part of its content refers to a research work which tries to comprehend if, in Argentina, state schools located in territories traumatically marked by inequality can produce educational experiences which alter the given order. It tries to explore, based on a school scene, the possibility to think school gestures from the perspective of movement and from the variation that takes place when a teaching team disputes the meanings of equality and it produces other ways to see childhood, childhoods. This is a text which explores the conditions of possibility in the light of the brutal advance of neoliberalism in our country. Boys and girls from the working-class are victims of "trigger-happy" police officers, of repression and persecution by security forces. Their educational careers are frequently interrupted by factors linked to the worsening of their life conditions. The infantilization of poverty in Latin American countries is reflected in the educational field. Segregated child populations attend devaluated school circuits which reproduce the exclusion processes. This paper tries to recover the educational experiences which interrupt what is expected, which dismantle the discourse of fear and insecurity and challenge the discursive equivalence that associates a poor boy or girl with a social danger. Its purpose is to put forth the idea that gestuality, institutional actions rooted in questions and movement which encourages thinking allow us to imagine other possible worlds, to write other melodies that vary from the expected and to invent other polyphonic textures in education. In other words, to cater for those small movements which, on the basis of equality, can define a direction in one way or another. Schools in working-class sectors frequently find themselves trapped in very complex processes; however, there are countless actions that, from the educational perspective, can be conducted to dispute the meanings of teaching in public terms.

keywords: school(s); educate; childhood(s); equality. 
la mariposa y el violín

la urgencia de una cita: infancia(s), escuela(s) e igualdad

\section{mariposas y violines}

Un día de clases, en una escuela de una barriada muy popular, uno de los grupos de alumnos/as de la mañana conserva en un frasco una oruga que ese día abandona la crisálida y se transforma en mariposa. Los chicos - según relatan sus maestros - se sorprenden por su transformación. No era una mariposa deslumbrante pero de todos modos los llena de asombro. En el patio, al compartir lo sucedido con otros, surge la pregunta sobre ¿qué hacer? La decisión final es dejarla en libertad para que vuele. En la escuela se encuentran los profesores y profesoras de la Orquesta ensayando los instrumentos con sus alumnos/as en las aulas. El día anterior, frente a la eventualidad y lo que provoca el nacimiento de una mariposa, el director de la escuela le consulta al director de la Orquesta si es posible sumar música para acompañar el primer vuelo. Todos se dirigen al patio. El director de la Orquesta improvisa con un violín una melodía y ese vuelo, mínimo, de una mariposa (común), en el patio de una escuela del conurbano, es acompañado por la música de un violín... Y se transforma en algo mágico, la mariposa y la música del violín embelesan a los que lo presencian. (Director, agosto de 2015, cit. por REDONDO, 2016, s.p.)

La mariposa nace en el patio rodeada de niños y niñas. Asimismo, el acompañar su nacimiento y primer vuelo por un violín nombra la novedad de la infancia. Envolver ese vuelo en música es un modo de nombrar, cuidar y acompañar otros posibles vuelos. Quizá, el de "los recién llegados", "los nuevos" al mundo. En continuidad con el pensamiento arendtiano, si la natalidad es un milagro y el nacimiento incluye la posibilidad de la continuidad, pero también de la discontinuidad de la humanidad, educar "es el modo como las personas, las instituciones, las sociedades responden a la llegada de los que nacen. La educación es la forma en que el mundo recibe a los que nacen. Responder es abrirse a la interpelación de una llamada y aceptar una responsabilidad". (ARENDT, cit. por LARROSA, 1998, p.73) ${ }^{2}$

¿Qué significa envolver con música el vuelo de la mariposa?

Las escuelas públicas que trabajan con infancias populares están habitadas por un sinfín de episodios cotidianos similares, pero estos suelen pasar inadvertidos al ser subsumidos por la descripción detallada de los entornos empobrecidos. Aquello que desde el discurso del poder enfatiza la negatividad de los sujetos es ponderado como lo que describe de manera total a quienes habitan en las barriadas populares, ello se derrama en la caracterización de las escuelas y sus estudiantes. Por tanto, quienes enseñan parecen quedar destinados/as y, de algún modo, sujetos/as a educar a una población descartable, un resto, como lo expresó una profesora en el

2 La traducción, cuya cita se encuentra en idioma original, es propia. 
marco de una investigación: "yo le enseño a la escoria, a lo más bajo entre lo más bajo" (MARTINIS y REDONDO, 2006, p.102).

En sentido contrario, en las experiencias educativas de carácter alternativo al modelo tradicional, distintos colectivos docentes traman otras metáforas en territorios marcados por el desamparo. La propuesta pedagógica se traduce en la vida cotidiana escolar como los acordes de una melodía que entrelaza sentidos educativos, perspectivas filosóficas y políticas que configuran una urdimbre que amarra, filia a los sujetos.

Los profesores y profesoras, los niños y niñas, los y las adolescentes, los grupos familiares, más todos aquellos adultos que componen la escuela, son actores y autores del acto educativo. Y de una invención cotidiana que altera lo dado en disputa con las biografías anticipadas. En definitiva, lo que acontece es que se articula de otro modo la relación entre la infancia, la(s) infancia(s) popular(es), la escuela, la pedagogía y la educación.

Estas formas integran una melodía que la escuela compone en un "entretiempo" junto con el entrelazamiento de los hilos de colores necesarios para cada tejido. Sin esa urdimbre/trama no hay construcción posible. Al pensar, el "hacer escuela" se estructura y desestructura.

Pero para nosotros, el acto principal y más importante que "hace escuela" tiene que ver con la suspensión de un presunto orden desigual. En otras palabras, la escuela ofreció tiempo libre, es decir, tiempo no productivo, a quienes por su nacimiento y por su lugar (por "su posición") no tenían derecho a reivindicarlo. (MASSCHELEIN, SIMONS, 2014, p.28)

La urdimbre de la "escuela de colores" amarra un tejido de sostén que la estructura, en la medida en que piensa, crea y hace. $Y$, en esa misma medida, al tomar las decisiones vinculadas a la enseñanza, se desestructura, con la misma plasticidad con la que un artesano talla su obra, una tejedora teje, un poeta rasga el papel con su poesía. ¿Maestros y maestras artesanas? ¿Maestros y maestras tejedoras? ¿Maestros y maestras que "urden" la trama de la igualdad y la diferencia?

Esta historia mínima de la mariposa y el violín marca huellas sutiles en la memoria y el relato de chicos/as y docentes. ¿Qué prevalece en ese instante? ¿La fugacidad de un momento único? ¿El contraste con el entorno social? ¿O la formación de la sensibilidad como textura imprescindible de todo proceso educativo?

Quizás el sentido lo hallemos en un acontecimiento profundamente verdadero que tiene como único fin la experiencia vivida... Las huellas del vuelo de la mariposa y los violines en barriadas desheredadas nos permiten pensar en las marcas de esta experiencia como "escritas" en la vida de sus protagonistas, con el único fin de afirmar más vida en la vida que están viviendo.

Es sugerente para nuestro análisis el pasaje de Infancia en Berlín, de Walter Benjamin, del chico frente a los calcetines de lana en el armario, que recupera Schèrer (2012, p. 58): 
Uno de los calcetines enrollados parecía estar contenido en la pequeña bolsa formada por otro. El niño hunde en ella su mano para agarrarlo y tira. Pero tan pronto como lo ha sacado de su bolsa, esta última desaparece. Experiencia verdaderamente filosófica de la infancia, en la que Benjamin dice haber descubierto, con la "aparición perturbadora" del calcetín desenrollado, "una verdad enigmática" que no dejará de acosarlo: "la forma y el contenido, la envoltura y lo envuelto, el calcetín de adentro y de la bolsa son una sola y misma cosa. Una sola cosa y una tercera también, es verdad: ese calcetín, fruto de su metamorfosis".

Schèrer, retomando esta vez a Gilbert Simondon, argumenta que lo viviente vive en el límite de sí mismo, sobre su límite, con una dirección hacia adentro y otra hacia afuera. Pone como ejemplo un tipo particular de membrana selectiva, una que permite la metamorfosis entre el interior y el exterior: la de la oruga luego mariposa (SIMONDON, cit. por SCHÈRER, 2012, p. 59). La mariposa y el violín; otra envoltura entre el interior y el exterior: la música.

La escuela especial envuelve a la infancia, y la cultura envuelve a la escuela. La escuela en-vuelve, vuelve adentro de la infancia. La cultura en-vuelve a la escuela: vuelve adentro de ella. La joven violinista y lutier, de liviana figura, ensaya en el parque o en la biblioteca, junto a sus alumnos y alumnas, niños y niñas, y también con madres del barrio. Relata:

Y cuando vine a vivir acá me fui a la capital ${ }^{3}$, entonces volver a salir de ahí es como tomar aire fresco por más que haya un viaje de una hora y pico; es como ver un poco más el cielo y por eso... Acá también, dar una clase en el pasto; antes dábamos clases allá en la huerta o acá mismo, ponemos la sillita... cuando está, ahora, la primavera. Y venir acá, tener las hamacas... es toda una experiencia muy linda. En este lugar, quién diría, uno entra y hay un mundo así, digamos... Después de una puerta podés entrar a cualquier lado; después de una puerta se pueden encontrar muchas cosas. Pero vos acá entrás y es como una cosa que no se puede creer, como que ves algo de una profundidad o de una amplitud, no sé bien cómo decirlo... (Profesora, septiembre de 2015, cit. por REDONDO, 2016, s.p.)

La existencia de un mundo que se profundiza o amplía nos invita a pensar en una escuela que va más allá de la escuela, que se abre al mundo... o que abre otros mundos para la escuela en la propia escuela.

En nuestra historia educativa latinoamericana, enseñar y aprender a partir de la expresión y la creación no es una novedad. Si alargamos la mirada hacia el pasado más próximo nos encontramos, por ejemplo, con la experiencia pedagógica del poeta-maestro uruguayo Jesualdo Sosa, que transcurre durante la década del treinta

\footnotetext{
${ }^{3}$ La profesora de violín se refiere a que, antes de trasladarse a la Ciudad de Buenos Aires, vivía en una ciudad del interior de la provincia homónima.
} 
en la escuela de las Canteras del Riachuelo, a una hora del puerto de Colonia en la República del Uruguay, donde asisten hijos de trabajadores inmigrantes de Europa del este, como rusos y checoslovacos, entre otros ${ }^{4}$. El maestro y pedagogo enseña en función de una propuesta curricular basada en la expresión creadora, que surge del convencimiento de que este concepto representa el camino para educar al niño en libertad y autonomía, a partir del propio niño y de su actividad 5 .

Pedagógicamente, se reconocen en Jesualdo las figuras del romanticismo revolucionario. Conduce en soledad una experiencia pedagógica de gran impacto en los discursos de liberación del niño; publica un libro acerca de ello, de fuerte tono pasional sobre las cuestiones humanas y sociales, sobre el sentido de la acción educadora (CARUSO, 1995, s.p.)

Asimismo, Luis Iglesias, maestro de la escuela rural bonaerense, es reconocido por su trabajo educativo y la creatividad de su propuesta pedagógica; entre otros, son famosas las exposiciones de acuarelas de sus alumnos y los poemas publicados. Autor de textos como el ensayo La escuela rural unitaria (1957), también contempla la belleza como parte indisoluble de la educación que lleva adelante y narra en su prolífica obra.

Hasta el día de hoy, las experiencias pedagógicas rurales del ámbito rioplatense continúan resonando en América Latina. Sin embargo, las políticas actuales en la Argentina propician el cierre de escuelas rurales, justificado por el dato cuantitativo de pocos estudiantes por aula presentado como una expresión de la inviabilidad de la educación pública en el campo.

El neoliberalismo en educación aggiorna en el siglo XXI su discurso y menosprecia la educación rural, la educación de personas adultas, la educación especial, los bachilleratos populares y aquellas formas de atención que no respondan al canon homogeneizador, $\mathrm{ni}$ al ajuste del gasto fiscal recomendado por los organismos internacionales y sus guarismos financieros traducidos como parámetros de calidad educativa. Un nuevo glosario eficientista, compuesto por un catálogo de clasificaciones y etiquetamientos de alumnas y alumnos al interior del sistema educativo público, incorpora un lenguaje tecnocrático y cientificista con el objeto de que sea transferido a la enseñanza sin mediaciones. El acto de educar como suspensión, y con la orientación a los y las alumnas hacia el mundo -más allá de su origen social- con el propósito de observar, experimentar, atender el interés del

\footnotetext{
${ }^{4}$ Véase Jesualdo Sosa, J. (1935). Vida de un maestro. Buenos Aires: Sociedad Amigos del Libro Rioplatense.

${ }^{5} \mathrm{Su}$ experiencia es reprimida por las autoridades educativas uruguayas, pero su repercusión internacional lo lleva a México entre 1938 y 1940, donde colabora en el gran terreno de experimentación de la "Educación socialista" llevado a cabo por el gobierno del Gral. Cárdenas (19341940). Allí ocupa la cátedra normalista en materias ligadas al universo de los niños, como la literatura infantil. Enseña la materia durante dos años en la Escuela Nacional de Maestros, y, a su regreso, obtiene la cátedra en la Universidad de la República; de todos modos, gran parte de su obra es publicada en la Argentina.
} 
sujeto por el mundo (MASSCHELEIN y SIMONS, 2014), que posibilita detener el tiempo escolar para capturar el vuelo de la mariposa se banaliza, en un intento de horadar el sentido público de la escuela.

\section{Urdimbre(s) escolar(es) y tejidos igualitarios}

Las escuelas públicas latinoamericanas, en particular, en los dos últimos años en Brasil y Argentina, se hallan atravesadas por procesos de mercantilización y privatización en el marco del retroceso y achicamiento de la educación pública. Políticas de ajuste, restricciones presupuestarias, campañas publicitarias que culpabilizan a los y las docentes por el supuesto retraso en los rankings internacionales, disciplinamiento ideológico y persecución política otorgan un carácter neoconservador a las medidas de los actuales gobiernos.

Ello se agrava en el caso de las escuelas populares que se desempeñan en espacios donde la pobreza y la exclusión se profundizan de manera acelerada. Los procesos de pauperización se producen bajo discursos piadosos y tecnocráticos que reordenan la tarea de profesoras y profesores en los espacios escolares orientándolas hacia tareas de precaria asistencia. Se suma un cambio curricular enmarcado en la educación emocional, que endiosa el aprendizaje y la calidad educativa para desplazar aceleradamente el lenguaje de la educación, la igualdad y la emancipación.

¿Cómo enfrentar el alud neoliberal en el día a día escolar?

A partir de la investigación y la propia experiencia de compartir tiempos y andares "entre" escuelas y espacios educativos afectados por la desigualdad, la mirada se detiene en los colectivos docentes más arraigados en la vida cotidiana de las escuelas públicas. No siempre se contempla lo que sucede en el imaginario de quienes cada día, día con día, asumen la enseñanza de las infancias populares. ¿Qué sentidos le otorgan a la enseñanza, la transmisión, la representación de otros mundos posibles? ¿Qué papel ocupa el pensar, caminar, construir e inventar con otros y otras? ¿De qué manera la escuela aloja a la infancia, la(s) infancia(s) popular(es)?

La expresión de los niños, niñas, adolescentes y jóvenes -tal como vemos a lo largo de las propuestas que se presentan- es un componente de la experimentación pedagógica. Sin embargo, esto no organiza una articulación homogénea en el discurso educativo de los y las docentes, pero sí sentidos pedagógicos comunes en el terreno de las prácticas al interior del equipo institucional, con las variaciones de los desempeños individuales y la experiencia acumulada a lo largo de los años.

Las escuelas y sus protagonistas, al igual que las tejedoras del Norte argentino, traman urdimbres, y sobre ellas dibujan sus diseños, que modifican en la medida que el movimiento que realizan colectivamente lo requiere. En la música, se crean texturas que de manera polifónica recrean melodías. En el medio del arrasamiento de los derechos de la infancia, el educar provoca un sutil desplazamiento, como el de una larva a mariposa acompañada por un violín en un patio escolar.

A pesar de que en la gran mayoría de las barriadas populares y comunidades se agravan las condiciones de vida y se acentúa la pérdida de la soberanía alimentaria de los grupos familiares, en el interior/exterior del espacio educativo un tejido igualitario se enlaza y entrama, uniendo los puntos del reconocimiento de un 
derecho -a la educación- y la apertura a todas las melodías que la humanización habilita.

\section{la partitura de la igualdad}

En tiempos tan desesperanzadores, ¿es posible pensar en una escuela pública que iguala?

Maestras y maestros, profesores y profesoras e infancias populares escriben una partitura compartida en la escuela investigada. Los elementos que la componen configuran una experiencia educativa con adolescentes y jóvenes de sectores populares que asisten a la institución al presentar una propuesta única en su forma y contenido en la educación especial de la provincia de Buenos Aires. Sus sentidos educativos más imperceptibles, aquellos que con suma frecuencia, pasan desapercibidos como saberes pedagógicos e institucionales se ponen en acto en esta y otras escuelas que atienden grupos populares. La clave: la construcción de preguntas, el fluir de un pensamiento colectivo y compartido, la interrupción de lo dado, el alojar la duda y la apertura a otras variaciones de lo posible. El "no saber" como la expresión cotidiana de un saber instituido e instituyente.

$\mathrm{Y}$, en los recorridos/caminos errantes, la sujeción a un principio -el de la igualdad de las mentes- y la confrontación argumentada y firme a las clasificaciones, a los etiquetamientos nuevos o viejos de la burocracia estatal y sus agencias.

La posición radical de igualar incluye la mirada y el gesto de alojar sin contemplaciones ni dobles discursos a todos los niños y niñas. Permitir el despliegue de lo (im)posible y hacer vibrar las notas de otros lenguajes y voces. ¿Será un requisito para las escuelas públicas de sectores populares escribir y reescribir las partituras escolares que, en sus variaciones, produzcan operaciones pedagógicas de verificación de la igualdad?

En ocasiones, quienes trabajan con infancias populares justifican la devaluación de las labores de enseñanza circunscribiéndolas a la instrucción o a resolver el cúmulo de tareas que demanda la reproducción del propio sistema educativo jerárquico, fragmentado y desigual. Se superponen una variedad de argumentos que reducen, simplifican y postergan la tarea de enseñar. Se le sobreimprime un discurso sobre el niño carente (MARTINIS y REDONDO, 2006) que adjudica a las dificultades del contexto la imposibilidad de educar, y el aumento de problemas sociales se cristaliza como fronteras educativas (REDONDO y THISTED, 1999).

El fracaso en las trayectorias escolares de las infancias populares se define por sus condiciones de vida de mayor vulnerabilidad, en vez de ser objeto de problematizaciones y desvelos para ser abordados desde la escuela. Se producen escenas inimaginables para otras escuelas públicas que atienden otros sectores sociales. El amor es ponderado como respuesta a una supuesta carencia, y un círculo vicioso reproduce aún más las condiciones de desigualdad como punto de partida. A los circuitos de escolarización y segmentación de la década de 1980 señalados por BRASLAVSKY (1985) a finales del siglo XX -y, agregamos, principios del siglo XXI- se suman aquellos indicados por las pedagogas Puiggrós y Dussel (1999, p. 16): 
El modelo neoliberal (...) produce una crisis orgánica del sistema educativo. Fractura los vínculos constitutivos de la organización político-institucional, de las diferencias generacionales y sociales y de la cadena de transmisión de la cultura. La escolarización no se dirige a la uniformización de toda la población, sino a su educación por bandas y sectores. El sistema escolar ha variado su función uniformizadora para tomar un rol protagónico en la producción de fronteras culturales.

La escuela pública se halla en el centro de la tormenta, los problemas estructurales que la disfuncionalizan no son resueltos en términos de las políticas necesarias. Frente a ello, maestras y maestros atienden cada día diversidad de tareas, casi todas los distancia de la especificidad de la enseñanza, que, en otras cosas, se rutiniza y banaliza. La pregunta que colectivos docentes se plantean, en algunos casos, es: ¿qué hacer en el mientras tanto?

La multidimensionalidad de la desigualdad (KESSLER, 2014) se reproduce en la vida cotidiana, la escuela abre sus puertas todos los días: ¿cómo enfrentar los efectos traumáticos de la exclusión en la vida de los y las estudiantes, las familias y los y las docentes?

La mariposa y el violín, como los espacios filosóficos en otras experiencias nacionales e internacionales, producen una música, quizá minimalista, sin gestos cargados de grandilocuencia que en la tarea cotidiana transcurren dentro y fuera de la escuela. Nombran y dan sentido al enseñar.

En los bachilleratos populares, en "la escuela de colores" ya citada, en las aulas que subvierten el orden de lo instituido, los muros escolares son perforados por la(s) cultura(s) y otra temporalidad, por viajes con grupos de niños y niñas, adolescentes y jóvenes que abren mundos a lo desconocido. Paradojalmente, incluso sin recursos, se parte a otros lugares, también dentro del espacio escolar. La vida no se limita a la reterritorialización que les propone el capitalismo a las infancias populares; por el contrario, se expande su experiencia de infancia a otros mundos. La vida escolar se amarra y se desamarra para viajar de todos los modos posibles. Las concepciones, los modos de ver y pensar la infancia, la educación y la igualdad representan la posibilidad del camino.

A pie, como las primeras familias, mujeres y hombres midieron las tierras que tomaron para construir un barrio de manera organizada, los niños y niñas experimentan salir de la escuela con su Orquesta Sinfónica a tocar en otros espacios. La diferencia es que, en vez de estar mendigando panes negados en estaciones y/o rutas, llevan en sus espaldas violines. O cargan violoncelos, flautas traversas, trombones y trompetas para llevar su música a otros territorios.

Ampliar los horizontes, pensar a cada chico y cada chica en su singularidad, otorgar lugar a su voz, sin diagnóstico en el caso de la escuela especial investigada, refleja otro vínculo entre la infancia, la educación y la pedagogía, pero sobre todo la igualdad. Los niños y niñas que en la Argentina actual son víctimas del gatillo fácil en las escuelas populares son reconocidos/as y valorados/as. La posición enseñante 
de sus profesores/as dialoga de algún modo con la que nos presenta el filósofo Walter Kohan al referirse al maestro Simón Rodríguez:

\begin{abstract}
Ese es tal vez el secreto de un buen maestro: mirar con los ojos bien abiertos a las niñas y los niños que lo observan. Acoger su mirada, atenderla, cuidarla, nutrirla, apreciarla. Enseñar es, ya lo sabemos, una cuestión de atención y sensibilidad (KOHAN, 2016, p. 64).
\end{abstract}

\title{
una cita urgente
}

Las escuelas que atienden grupos populares en la Provincia de Buenos Aires quedan distantes de los centros urbanos; metáforas que las localizan en el fondo informan de desplazamientos de profesores y profesoras, maestros y maestras para poder llegar.

El mapa de los lugares de encuentro y los itinerarios compartidos se entraman con los de otros/as docentes que viven en La Matanza; quienes tienen transporte propio llevan a quienes no lo tienen, a quienes viven cerca entre sí o a quienes que se acercan hasta el camino acordado. Profesores y profesoras, integrantes de la Orquesta, se citan y entrelazan sus recorridos para llegar. Viajan de a uno, de a dos, de a tres, de a cuatro, para acercarse hasta la institución que se constituye en un lugar de encuentro. La distancia recorrida varía para unos y otros, pero es significativa para todos. Llegar o salir de la escuela implica un esfuerzo desde cualquier punto geográfico, incluso para quienes viven más cerca, debido a la dificultad que representa la escasa frecuencia de los medios de transporte que ingresan a los barrios de Villa Scasso.

A partir de estas "citas de maestros y maestras" que inician el día escolar extramuros de la escuela, a primera hora se comienzan a esbozar las primeras preguntas. En los trayectos se entrecruza e intercambia información necesaria para la organización de las actividades, se arman propuestas e impresiones sobre situaciones escolares, se exponen las novedades y se preparan las sorpresas del día. ¿Dónde empieza la escuela? ¿La escuela comienza antes de la escuela?

Durante los viajes conversan un mix de cuestiones: de género, de las vivencias personales que se mezclan con la vida de la escuela, las tareas por resolver ese día $u$ otro. Para quienes asumen una posición clara y cuidadosa respecto de la niñez con la cual trabajan, ese tiempo es un tiempo de bordado. De repasar los puntos dados, los pendientes y las posibilidades futuras de cada alumna y alumno a pesar de aquello que incide directamente en las trayectorias escolares para negar el porvenir.

Es decir, bordar junto con cada niño, niña, adolescente, joven un destino diferente al que le designan las fuerzas de seguridad y los medios de comunicación. Un bordado antidestino (NÚÑEZ, 2005) que se da en los viajes magisteriales hacia las escuelas al inicio y al cierre de la jornada, donde las y los docentes asumen una cita con el tiempo y la esperanza.

Asimismo, se constituye un nos-otros que refiere a quienes conforman la escuela, pero no de forma endogámica: por el contrario, es un nos-otros que se altera, modifica su contorno y se expande en muchas ocasiones; por ejemplo, cuando la escuela "hace escuela" fuera del cerco escolar, en el propio barrio, en las casas de las 
familias de los y las estudiantes -tanto de quienes ya son parte de la institución como de los/as que serán admitidos/as a futuro-.

Ese nos-otros también se amplía con la presencia de lo "extranjero" que ingresa a la escuela o que la escuela sale a buscar: se entrelaza una dimensión temporal y un espacio diferente, que yuxtapone el tiempo chrónos, equivalente al tiempo escolar tradicional, lineal y sucesivo, con otro, el tiempo aión, término que "designa la intensidad del tiempo de la vida, una duración, un destino, algo así como una temporalidad no numerable, ni consecutiva ni sucesiva" (KOHAN, 2014, p. 95) y que se liga a la posibilidad de la experiencia.

En la escuela investigada los espacios se habitan, los techos de las aulas brillan con las estrellas de Van Gogh, en la huerta se cosechan hortalizas y se plantan fresias, los artistas pintan murales con niños, niñas, maestras y maestros, una playa se recrea en el patio y se construye una cascada. Las gárgolas que se crean se eligen con los niños y niñas, los libros vuelan en la biblioteca, o se leen en bancos para tener los pies en el aire, el día dedicado a México se transforma en una escenografía mexicana. Frida Kahlo llega de visita. Otros mundos ingresan al mundo escolar.

Un sinfín de propuestas le otorga un ritmo cotidiano a la escuela, intenso y sostenido. Cada día la comunidad es parte del asombro y la invención del hacer educativo cotidiano.

La escuela popular asume un modo propio de recibir y alojar a la(s) infancia(s), como así también, una manera de sostener la enseñanza y la transmisión que define una posición enseñante, y un acto político impensado en barrios extremadamente empobrecidos: el de dibujar un territorio donde irrumpa y acontezca la igualdad y la emancipación.

\section{últimas pinceladas}

Educar en barrios populares significa, ante todo, atravesar cada día corporalmente el estigma. Ingresar/transitar/ser parte de un mundo marcado por la segregación y la exclusión.

Educar en barrios populares significa transformar la precariedad en un acto de resistencia.

Educar en barrios populares implica presentarse a una cita. Una cita con la infancia, la(s) infancia(s), en el presente y el porvenir.

Educar en barrios populares representa un movimiento, en ocasiones imperceptible incluso para quienes lo protagonizan, un movimiento... leve y potente. Un movimiento sutil como el vuelo de una mariposa o el sonido de un violín.

\section{referencias}

BRASLASVSKY, C. La discriminación educativa en Argentina. Buenos Aires: FLACSO - GEL. 1985. 
CARUSO, M. Pedagogía, currículum e intelectuales: El caso de las izquierdas argentinas ante el primer peronismo (1943-1955). Informe final Programa de Becas de Investigación. Buenos Aires: Universidad de Buenos Aires. 1995.

KESSLER, G. Controversias sobre la desigualdad: Argentina, 2003-2013. Buenos Aires: Fondo de Cultura Económica. 2014.

KOHAN, W. O. Infancia. Entre educación y filosofía. Barcelona: Laertes. 2004.

. El maestro inventor: Simón Rodríguez. Caracas: Ediciones del Solar. 2016.

IGLESIAS, L. La escuela rural unitaria: Fermentario para una pedagogía creadora. Buenos Aires: Ed. Magisterio del Río de la Plata. 1995.

LARROSA, J. Imagens do outro. Petrópolis: Vozes. 1998.

MARTINIS, P. y REDONDO, P. (comp.). Igualdad y educación. Escrituras entre (dos) orillas. Buenos Aires: Del Estante. 2006.

MASSCHELEIN, J. y SIMONS, M. Defensa de la escuela: Una cuestión pública. Buenos Aires: Miño y Dávila. 2014.

NÚÑEZ, V. "El vínculo educativo". En H. Tizio (coord.), Reinventar el vínculo educativo: Aportaciones de la Pedagogía Social y el Psicoanálisis (pp.19-48). Barcelona: Gedisa. 2005.

PUIGGRÓS, A. y DUSSEL, I. “Fronteras educativas en el fin de siglo: Utopías y distopías en el imaginario pedagógico". En En los límites de la educación: Niños y jóvenes del fin de siglo (pp. 7-23). Rosario: Homo Sapiens. 1999.

REDONDO, P. La escuela con los pies en el aire. Hacer escuela entre la desigualdad y la emancipación. Tesis doctoral, FaCHE-UNLP. 2016. . y THISTED, S. Las escuelas primarias "en los márgenes". Realidades y futuro. En A. Puiggrós, En los límites de la educación: Niños y jóvenes del fin de siglo. Rosario: Homo Sapiens. 1999.

SCHÉRER, R. Infantis: Charles Fourier e a infância para além das crianças. Belo Horizonte: Autêntica. 2009.

recibido en: 21.07.2018 aprobado en: 18.08.2018 